\title{
Uso de diferentes pontas de pulverização no controle de doenças no trigo
}

\author{
Using different spray tips to control diseases in wheat
}

\author{
Alicia Baumhardt Dorneles ${ }^{1}$, Eduardo Leonel Bottega ${ }^{2}$, Zanandra Boff Oliveira ${ }^{3}$, \\ Alberto Eduardo Knies ${ }^{4}$, Clarissa Moraes da Silva ${ }^{5}$ e Irajá Jantsch de Souza ${ }^{6}$ \\ 1,2,3,5,6 Universidade Federal de Santa Maria - Campus Cachoeira do Sul, RS, Brasil \\ alicia_dorneles@outlook.com eduardo.bottega@ufsm.br ; zanandraboff@gmail.com; clarissamoraes37@outlook.com; \\ iraja_14@yahoo.com.br \\ ${ }^{4}$ Universidade Estadual do Rio Grande do Sul, Cachoeira do Sul, Brasil \\ albertoek@gmail.com
}

\section{Resumo}

Fatores ambientais instáveis auxiliam na incidência de doenças e pragas, causando redução da produção e a contaminação do grão, destacando-se o ataque por Giberela (Fusarium graminerum). Neste cenário, se faz necessário o uso de técnicas de manejo adequadas. Estratégias como escolha da adequação da ponta de pulverização no controle químico são indispensáveis. Sendo assim, este trabalho teve como objetivo estudar o emprego de quatro pontas de pulverização: CVI 11002, ADI ISO 11002, TVI ISO 8002 e ATR 8002. O experimento foi conduzido na área experimental da Universidade Estadual do Rio Grande do Sul, no distrito de Três Vendas, em Cachoeira do Sul/RS. A cultivar utilizada foi TBIO SELETO. O fungicida empregado foi o Nativo ${ }^{\circledR}$. Foi adotado o delineamento experimental inteiramente ao acaso com 4 repetições e parcela testemunha. Foram avaliados os seguintes parâmetros: peso de mil grãos (PMG, \%), peso hectolitro (PH; $\mathrm{kg} \mathrm{hL}^{-1}$ ) e produtividade (PRD; $\mathrm{kg} \mathrm{ha-}$ $\left.{ }^{1}\right)$. A variância foi testada aplicando-se o teste $F$ de Snedcor $(p<0,05)$ e as médias foram comparadas pelo teste de médias de Tukey ( $<<0,05)$. O peso de mil grãos, o peso hectolitro e a produtividade não foram influenciados pela utilização de diferentes pontas de pulverização adotadas no controle fitossanitário na cultura do trigo.

Palavras-chave: Triticum spp; Fusarium graminerum; Tecnologia de aplicação

\section{Abstract}

Unstable environmental factors contribute to the incidence of diseases and pests, causing reduced yield and grain contamination, especially the attack by Giberela (Fusarium graminerum). In this scenario, appropriate management techniques are needed. Strategies such as choosing the suitability of the spray tip for chemical control are indispensable. The aim of this study was to use four spray tips: CVI 11002, ADI ISO 11002, TVI ISO 8002 and ATR 8002. The experiment was conducted in the experimental area of The State University of Rio Grande do Sul, in the district of Três Vendas, in Cachoeira do Sul-RS. The cultivar used was TBIO SELETO. The fungicide used was Nativo ${ }^{\circledR}$. A completely randomized experimental design with 4 replicates and a control plot was adopted. The following parameters were evaluated: weight of one thousand grains (PMG, \%), hectoliter weight

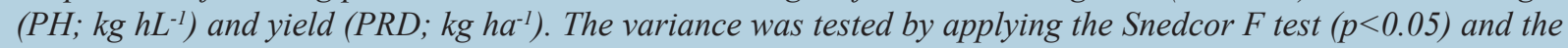
means were compared by the Tukey's test $(p<0.05)$. The weight of one thousand grains, hectoliter weight and yield were not influenced by the use of different spray tips adopted in the wheat phytosanitary control. 


\section{Introdução}

O trigo (Triticum spp.) é atualmente considerado um dos mais importantes cereais produzidos, constituindo parte considerável da economia e produção agrícola mundial, estando presente na alimentação animal e humana. A área cultivada atualmente com esta cultura ocupa cerca 1.928,1 hectares, sendo a região Sul do país a responsável pela maior parte de toda área com 1715,7 hectares (CONAB, 2017). A estimativa de produção para safra de 2017, de acordo com a Companhia Nacional de Abastecimento (CONAB, 2017), é de 5.221,4 toneladas.

Com amplo cultivo na região Centro-Oeste, Sudeste e Sul do Brasil, a produtividade do trigo depende de fatores inconstantes relacionados ao meio ambiente (em especial ao clima) e às técnicas de manejo empregadas na cultura. Portanto, o conhecimento da cultura, suas exigências, técnicas aplicadas ao controle de pragas e doenças é fundamental para que sejam alcançados bons resultados produtivos (MAPA, 2015).

A giberela está entre as doenças de maior importância mundial na cultura do trigo. No Brasil, é causada principalmente por espécies do complexo Fusarium graminearum, com destaque para $F$. graminearum sensu stricto [ou simplesmente F. graminearum (Fgss)] e F. meridionale (Fmer) (SCOZ et al., 2009; ASTOLFI et al., 2012). Os danos ocasionados por giberela em trigo no Brasil incluem a redução no rendimento e a contaminação com as micotoxinas de desoxinivalenol (DON) e nivalenol (NIV) (DEL PONTE et al., 2012). Segundo Casa; Bogo; Moreira (2007), a doença pode causar danos médios de $18,62 \%$ e no máximo $30,8 \%$. Por isso, esforços devem ser redobrados na busca da melhora da eficiência do controle. Devido aos prejuízos causados pela giberela, o uso de práticas de manejo adequadas são de grande valia para o sucesso da produção. Dentre estas práticas, destaca-se e o controle químico da doença, a partir do emprego de fungicidas (CUNHA et al., 2001).

A tecnologia de aplicação é empregada para melhorar a eficiência no uso de produtos fitossanitários. Uma aplicação eficiente é aquela onde ocorre deposição do produto no alvo biológico, reduzindo as perdas para o ambiente. Alguns fatores como a pressão empregada, o volume de calda aplicado e as pontas de pulverização utilizadas representam papel fundamental na eficiência do produto usado no controle das diversas moléstias, estando estes fatores diretamente relacionados com o tamanho de gota.

O uso de diferentes pontas de pulverização para obtenção de melhor controle de doenças já obteve resultado satisfatório quando aplicado à ferrugem asiática da soja, onde pontas do tipo cone proporcionaram a penetração de maior número de gotas com menor diâmetro médio volumétrico (FAVELLA, 2012). Para a cultura do algodão, o aumento ou diminuição do tamanho da gota melhora a eficiência da pulverização sob condições consideradas adversas, onde pontas de jato plano com indução de ar demonstraram maior volume de deposição nas folhas e maior uniformidade de pulverização em diferentes níveis da planta, o que acaba por favorecer o controle de possíveis moléstias (SOUZA; CASTRO; PALLADINI, 2007).

As mais variadas culturas já fazem uso de novas tecnologias de aplicação em busca de maior produtividade e qualidade, onde as pontas de pulverização possuem papel de extrema importância para alcançar os objetivos esperados. O tamanho das gotas produzidas pelas pontas determina a distribuição do agroquímico e o molhamento da planta para que o alvo em questão seja atingido (MENEGHETTI, 2006). Sendo assim, este trabalho teve por objetivo avaliar o efeito do uso de diferentes pontas de pulverização utilizadas no controle de giberela no trigo. 


\section{Materiais e métodos}

O experimento foi conduzido na área experimental da Universidade Estadual do Rio Grande do Sul, localizada a $29^{\circ} 53^{\prime}$ 00" de latitude Sul e a 530 00'00" de longitude Oeste, no distrito de Três Vendas, em Cachoeira do Sul/RS. A altitude média da região é de 125 metros. O clima da região é classificado como Clima subtropical, com verão quente (Cfa). A precipitação média anual varia de 1600 a $1900 \mathrm{~mm}$, com temperatura média anual de $20^{\circ} \mathrm{C}$ (ALVARES et al., 2013). O solo da área experimental é classificado como ARGISSOLO VERMELHO (EMBRAPA, 2013).

A semeadura foi realizada no dia 15 de maio de 2017, de forma manual. Foi utilizado um rastel de fabricação artesanal para abertura das linhas, equipado com 05 hastes espaçadas em 0,17 m. A cultivar utilizada foi TBIO SELETO. Foram distribuídas 330 sementes aptas por metro de linha. Após a semeadura, foi aplicado $250 \mathrm{~kg}^{-1}$ de adubo na formulação 09-33-12 (NPK) em cobertura. Quando a cultura atingiu o estádio de perfilhamento pleno, foi realizada adubação nitrogenada de cobertura, aplicando-se $180 \mathrm{~kg}$ de ureia (45\% de N), totalizando um fornecimento de 103,5 kg de nitrogênio por hectare. Foi adotado o delineamento experimental inteiramente ao acaso (DIC) com 4 repetições. As pontas de pulverização estudadas foram as seguintes: CVI 11002 (leque com indução de ar); ADI ISO 11002 (leque padrão); TVI ISO 8002 (cone vazio com indução de ar) e ATR 8002 (cone vazio). Conduziu-se também quatro parcelas como testemunha, as quais não receberam aplicações de fungicida.

O experimento ocupou área de $61,2 \mathrm{~m}^{2}$, onde foram alocadas 20 parcelas experimentais. Cada parcela experimental ocupou área de 3,06 $\mathrm{m}^{2}$, sendo 1,53 metros de largura (9 linhas espaçadas em 0,17 m) por 2,0 metros de comprimento. A área útil considerada nas avaliações foi de $1,19 \mathrm{~m}^{2}$, resultante do descarte de 0,5 metros de cada extremidade das parcelas e das duas linhas marginais (7 linhas x 0,17 $\mathrm{m} \times 1 \mathrm{~m})$.

O fungicida empregado no controle da giberela foi o Nativo® (TRIFLOXISTROBINA + TEBUCONAZOL), na dose de $0,75 \mathrm{~L}$ de produto comercial por hectare, conforme recomendação do fabricante, prescrita na bula do produto. As aplicações foram iniciadas na fase de florescimento pleno, sendo a primeira no final desta fase e a segunda 15 dias após a primeira. Para as aplicações do fungicida, foi utilizado um equipamento de pulverização costal de precisão, pressurizado com $\mathrm{CO}_{2}$ e barra contendo dois bicos espaçados entre si em $0,40 \mathrm{~m}$. Foi empregado volume de aplicação de calda de $150 \mathrm{~L}$ ha ${ }^{-1}$ e as pressões utilizadas foram de 75 PSI para as pontas do tipo cone e 30 PSI para as pontas do tipo leque.

Foram avaliados os seguintes parâmetros: incidência da doença (I, \%), severidade da doença (S, \%), peso de mil grãos (PMG, \%), peso hectolitro ( $\left.\mathrm{PH} ; \mathrm{kg} \mathrm{hL}^{-1}\right)$ e produtividade (PRD; $\mathrm{kg} \mathrm{ha}^{-1}$ ). Para as avaliações de incidência, severidade e peso hectolitro, foram colhidas 200 espigas por tratamento ( 25 espigas por parcela). O peso hectolitro foi determinado utilizando aparelho portátil de determinação de umidade e peso hectolitro da marca Agrologic $®$. A incidência e severidade da doença foram avaliadas por meio de coleta aleatória de espigas nas três linhas centrais de cada parcela em estado de grão mole, estádio 85 da escala sugerida por Zadoks (ZADOKS et al., 1974). Para avaliação de severidade foi utilizada escala diagramática sugerida por Stack; McMullen (2011). O peso de mil grãos foi obtido pesando mil grãos. A produtividade foi obtida pesando a massa de grãos colhidos em $1,19 \mathrm{~m}^{2}$, sendo o valor extrapolado para $10.000 \mathrm{~m}^{2}$ (1 hectare).

Os dados foram digitados em uma planilha Excel, que serviu como banco de dados para as análises estatísticas. A normalidade dos dados foi testada aplicando-se o teste de normalidade de Shapiro-Wilk $(\mathrm{p}<0,05)$. A variância foi testada aplicando-se o teste $\mathrm{F}$ de Snedcor $(\mathrm{p}<0,05)$ e as médias comparadas pelo teste de médias de Tukey $(\mathrm{p}<0,05)$. O programa estatístico utilizado foi o STATISTICA ${ }^{\circledR}$ versão 7 (STATSOFT, Inc. 2004). 


\section{Resultados e discussão}

Não foi observado ataque de $F$. graminearum, fungo causador da giberela, por este motivo não foi possível estimar a incidência e severidade da doença, mesmo no tratamento testemunha, onde não foi realizada aplicação de fungicida. A não ocorrência da doença pode ser explicada por três fatores: 1) Falta de condições climáticas para desenvolvimento do fungo, 2) Inexistência de inóculo da doença na área de estudo e 3) Resistência da cultivar de trigo ao ataque do fungo, que, segundo a empresa BIOTRIGO, apresenta resistência moderada.

$\mathrm{Na}$ Tabela 1 é apresentado o resumo estatístico das variáveis estudadas. Pode-se observar que o peso hectolitro teve médias entre 50 a $59 \mathrm{~kg} \mathrm{hL}^{-1}$ nos diferentes tratamentos, valores que ficaram abaixo do encontrado por Mafalda (2012), Gulart et al. (2013), Ormond et al. (2013) e Tonin (2011), que variam de 69 e $80 \mathrm{~kg} \mathrm{hL}^{-1}$. De acordo com a Normativa $\mathrm{n}^{\circ} 38$ de 30 de novembro de 2010 do Ministério da Agricultura, Pecuária e Abastecimento (Brasil, 2011), os grãos colhidos nos diferentes tratamentos estão na classificação "Fora de Tipo" para a moagem e outras finalidades por apresentarem valores menores que $72 \mathrm{~kg} \mathrm{hL}^{-1}$.

Tabela 1- Estatística descritiva para o valor do peso hectolitro $\left(\mathrm{PH}, \mathrm{kg} \mathrm{hL}^{-1}\right)$, peso de mil grãos (PMG, g) e produtividade (PRD, $\mathrm{kg} \mathrm{ha}^{-1}$ ), obtidos em função do tratamento com fungicida utilizando diferentes pontas de pulverização. Cachoeira do

$\mathrm{Sul} / \mathrm{RS}, 2017$

\begin{tabular}{|c|c|c|c|c|c|}
\hline & Mínimo & Média & Máximo & Desvio Padrão & $\mathrm{CV}(\%)$ \\
\hline \multicolumn{6}{|c|}{ Peso hectolitro $\left(\mathrm{kg} \mathrm{hL}^{-1}\right)$} \\
\hline CVI & 35,70 & 57,13 & 73,50 & 15,79 & 27,65 \\
\hline ADI & 36,90 & 49,10 & 57,60 & 8,98 & 18,29 \\
\hline ATR & 30,80 & 52,55 & 73,50 & 19,38 & 36,88 \\
\hline TVI & 31,20 & 52,43 & 71,00 & 16,51 & 31,49 \\
\hline Testemunha & 58,00 & 58,25 & 58,50 & 0,29 & 0,50 \\
\hline \multicolumn{6}{|c|}{ Peso de mil grãos (g) } \\
\hline CVI & 42,00 & 43,00 & 44,00 & 0,82 & 1,90 \\
\hline ADI & 43,00 & 44,25 & 45,00 & 0,96 & 2,16 \\
\hline ATR & 42,00 & 43,50 & 46,00 & 1,73 & 3,98 \\
\hline TVI & 40,00 & 43,75 & 46,00 & 2,87 & 6,57 \\
\hline Testemunha & 41,00 & 43,00 & 45,00 & 2,31 & 5,37 \\
\hline \multicolumn{6}{|c|}{ Produtividade $\left(\mathrm{kg} \mathrm{ha}^{-1}\right)$} \\
\hline CVI & 2517,25 & 3526,08 & 4304,31 & 743,64 & 21,09 \\
\hline ADI & 2604,71 & 3193,18 & 3569,41 & 423,15 & 13,25 \\
\hline ATR & 2279,02 & 3341,27 & 4384,22 & 948,93 & 28,40 \\
\hline TVI & 2349,10 & 3316,66 & 4255,33 & 791,15 & 23,85 \\
\hline Testemunha & 3558,53 & 3597,00 & 3635,47 & 44,42 & 1,23 \\
\hline
\end{tabular}


Trabalhos realizados por Panisson et al. (2003) e Ormond et al. (2013) resultaram em médias entre 31,7 e 35,12 gramas de mil grãos de diferentes cultivares de trigo. A BIOTRIGO Genética, através de dois anos de estudos realizados por Tonin et al. (2011), estipularam para a cultivar TBIO Seleto uma média entre 35 a 36 gramas. Sendo assim, é possível constatar que as médias observadas em relação ao peso de mil grãos, em função do tratamento com diferentes pontas, assim como o observado para a testemunha, está acima do padrão estipulado com valores de pouca diferença e coeficientes de variação que confirmam a baixa dispersão entre os dados.

Na Figura 1 são apresentados os gráficos de distribuição de frequência e resultado do teste de normalidade para as variáveis: peso hectolitro (Fig. 1a); peso de mil grãos (Fig. 1b) e produtividade de grãos (Fig. 1c). De acordo com o teste de normalidade de Shapiro-Wilk $(\mathrm{p}<0,05)$, todas as variáveis apresentam distribuição normal, o que é uma das premissas para a aplicação da análise de variância pelo teste F de Snedcor.

Figura 1 - Gráficos de distribuição de frequência das variáveis: peso hectolitro (a); peso de mil grãos (b) e produtividade de grãos (c)
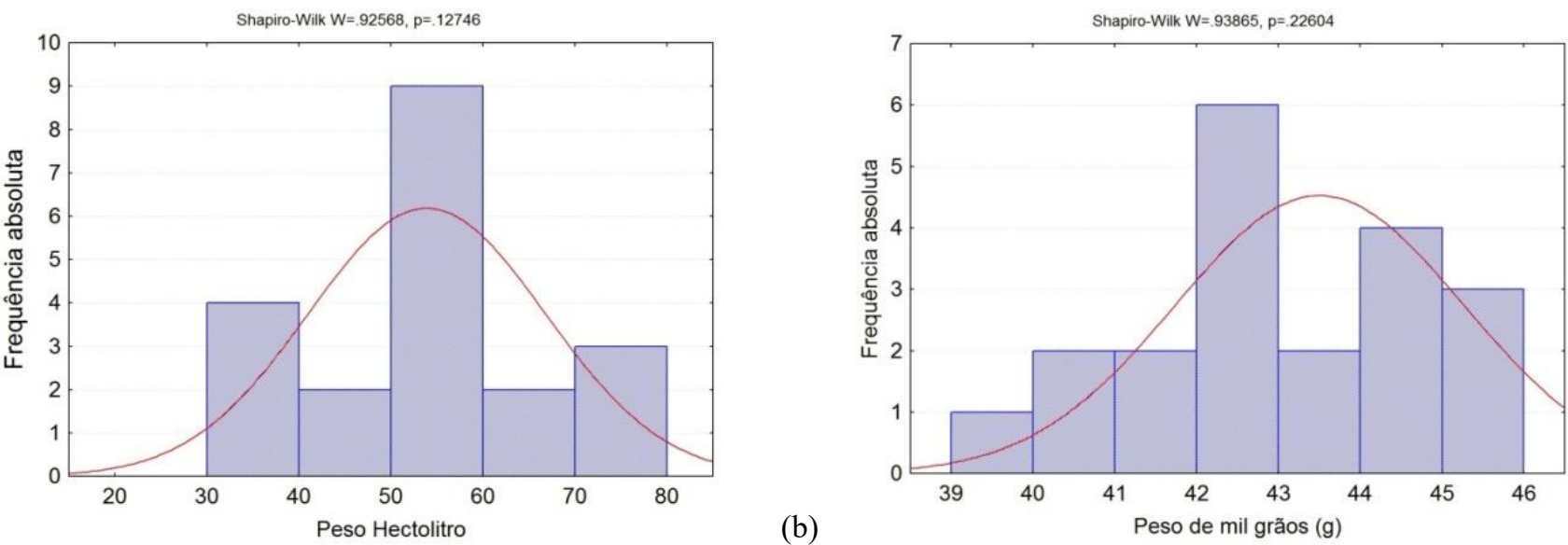

(a)

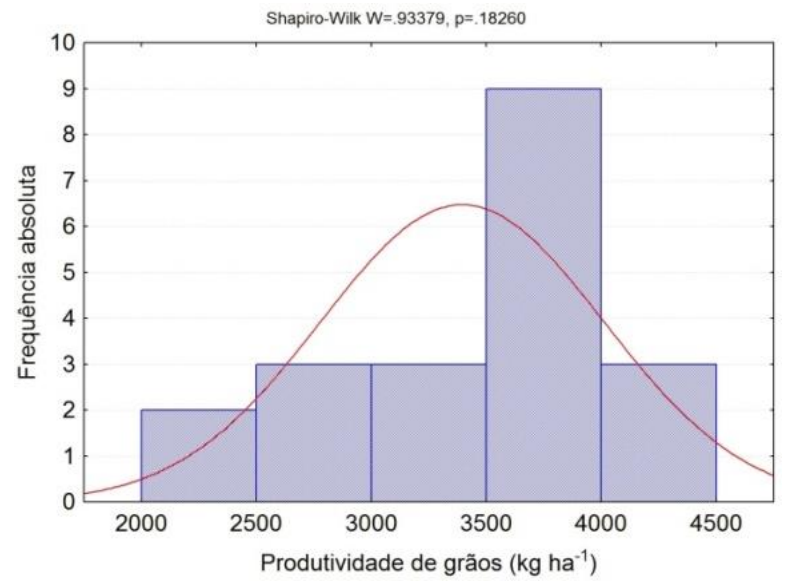

(c)

Na Tabela 2 são apresentados os resultados da análise de variância para os valores de peso hectolitro $\left(\mathrm{PH}^{\mathrm{kg}} \mathrm{hL}{ }^{-1}\right)$, peso de mil grãos (PMG, g) e produtividade (PRD, kg ha ${ }^{-1}$ ). De acordo com o teste $\mathrm{F}$ de Snedcor ( $\left.\mathrm{p}<0,05\right)$, não foi observada diferença significativa para as variáveis estudadas, demostrando que os fatores de variação (FV) não influenciaram as médias destas variáveis. O coeficiente de variação $(\mathrm{CV})$ obtido para o peso de hectolitro $(\mathrm{CV}=25,96 \%)$ demonstra precisão regular 
dos dados e é classificado como alto (CV entre 20 e 30\%), ótima precisão dos dados e CV classificado como baixo foi obtido para o peso de mil grãos $(\mathrm{CV}=4,38 \%)$, o $\mathrm{CV}$ obtido para produtividade $(\mathrm{CV}=19,81 \%)$ é classificado como médio e demonstra boa precisão dos dados (CV entre 10 e 20\%), de acordo com classificação proposta por Ferreira (1991).

Tabela 2 - Resultado da análise de variância para os valores de peso hectolitro $\left(\mathrm{PH}, \mathrm{kg} \mathrm{hL}^{-1}\right)$, peso de mil grãos (PMG, g) e produtividade (PRD, $\left.\mathrm{kg} \mathrm{ha}^{-1}\right)$. Cachoeira do Sul/RS, 2017

\begin{tabular}{|c|c|c|c|c|c|}
\hline \multicolumn{6}{|c|}{ Peso hectolitro (PH, $\left.\mathrm{kg} \mathrm{hL}^{-1}\right)$} \\
\hline FV & GL & SQ & QM & $\mathrm{Fc}$ & $\operatorname{Pr}>\mathrm{Fc}$ \\
\hline PONTAS & 4 & 225,44 & 56,36 & 0,28 & 0,88 \\
\hline Erro & 15 & 2934,73 & 195,64 & & \\
\hline Total corrigido & 19 & 3160,17 & & & \\
\hline CV $(\%)$ & 25,96 & & & & \\
\hline Média geral: & 53,89 & & & & \\
\hline \multicolumn{6}{|c|}{ Peso de mil grãos (PMG, g) } \\
\hline PONTAS & 4 & 4,5 & 1,12 & 0,31 & 0,86 \\
\hline Erro & 15 & 54,5 & 3,63 & & \\
\hline Total corrigido & 19 & 59,00 & & & \\
\hline CV $(\%)$ & 4,38 & & & & \\
\hline Média geral: & 43,50 & & & & \\
\hline \multicolumn{6}{|c|}{ Produtividade (PRD, $\mathrm{kg} \mathrm{ha}^{-1}$ ) } \\
\hline PONTAS & 4 & 430968,94 & 107742,23 & 0,23 & 0,91 \\
\hline Erro & 15 & 6781275,59 & 452085,03 & & \\
\hline Total corrigido & 19 & 7212244,54 & & & \\
\hline CV $(\%)$ & 19,81 & & & & \\
\hline Média geral: & 3394,83 & & & & \\
\hline
\end{tabular}

Número de observações: 20

\section{Conclusão}

O peso hectolitro, o peso de mil grãos e a produtividade não foram influenciados pela utilização de diferentes pontas de pulverização adotadas no controle fitossanitário da giberela, nas condições deste estudo. Recomenda-se a repetição desta pesquisa para confirmação dos resultados observados.

\section{Agradecimentos}

Os autores agradecem à Universidade Federal de Santa Maria pela concessão da bolsa de estudos através do Edital FIPE ARD 2017. Também são gratos à Universidade Estadual do Rio Grande do Sul (UERGS) pela disponibilização da área experimental. 


\section{Referências}

CASA, R. T.; BOGO, A; MOREIRA, E. Época de aplicação e desempenho de fungicidas no controle da giberela em trigo.

Ciência Rural, Santa Maria, v.37, n.6, p.1558-1563, 2007.

CONAB - Companhia Nacional de Abastecimento. Acompanhamento da safra brasileira de grãos 2016-2017. Brasília, v. 4, n. 12, p. 1-158, 2017. Disponível em:https://www.conab.gov.br/OlalaCMS/uploads/arquivos/17_09_12_09_01

_56_boletim_graos_setembro_2017.pdf Acesso em: 16 março 2017.

DEL PONTE, E.M. et al. Deoxynivalenol and nivalenol in commercial wheat grain related to Fusarium head blight epidemics in southern Brazil. Food Chemistry, v.132, p.1087-1091, 2012.

FERREIRA, P.V. Estatística experimental aplicada à agronomia. Maceió, EDUFAL, 1991. 440p.

MAPA - MINISTÉRIO DA AGRICULTURA, PECUÁRIA E ABASTECIMENTO. Cultura do trigo. Brasília/DF, 2015.

Disponível em: http://www.agricultura.gov.br/vegetal/culturas/trigo Acesso em: 16 março 2017.

MENEGHETTI, R. C. Tecnologia de aplicação de fungicidas na cultura do trigo. Dissertação de Mestrado, 58p. Santa Maria, Rio Grande do Sul, 2006. Disponível em: http://cascavel.cpd.ufsm.br/tede/tde_busca/arquivo.php?codArquivo =2157 Acesso em: 16 março 2017.

SOUZA, R. T.; CASTRO, R. D.; PALLADINI, L. A. Depósito de pulverização com diferentes padrões de gotas em aplicações na cultura do algodoeiro. Revista Engenharia Agrícola, Jaboticabal, v.27, p.75-82, jan. 2007.

STACK, R. W.; McMULLEN, M. P. A visual scale to estimate severity of Fusarium head blight in wheat. North Dakota State University, NDSU Extension Service, PP-1095. 2p. 2011.

STATSOFT, Inc. (2004). STATISTICA (data analysis software system), version 7. www.statsoft.com.

ZADOKS, J. C.; CHANG, T. T.; KONZAK, C. F. A decimal code for the growth stages of cereals. Weed Research, Oxford, v. 14, n. 1, p. 415-421, 1974.

PANISSON, E., et al. Técnicas de aplicação de fungicida em trigo para o controle de giberela (Gibberella zeae) ${ }^{1}$. Ciência Rural, Santa Maria, v.33, n.1, p. 13-20, jan-fev 2003. 